\title{
Theoretical Basis For A New Development Rate Model For Positive Photoresists
}

\author{
Ralph R. Dammel \\ AZ Photoresist Products, Hoechst Celanese Corporation, Somerville, New Jersey, USA
}

\begin{abstract}
A new development rate equation is proposed which is based on an equation derived by Huang, Reiser and Kwei for the concentration dependence of the dissolution rate of acidic resins in aqueous alkaline developers. This equation predicts cessation of development at a critical concentration $c^{*}$. An S-shaped function is proposed for the dependence of the critical concentration $c^{*}$ on the normalized sensitizer concentration $\mathrm{m}$ of positive-tone resists, and the model is tested with good success on a high speed and a high resolution $i$-line photoresist.
\end{abstract}

Keywords: enhanced Mack model, Huang-Reiser-Kwei equation, AZ resist, development parameters

\section{Introduction}

The exposure step in photolithography, complete with changes in absorbance and standing waves, is well described by the $\mathrm{A}, \mathrm{B}$, and $\mathrm{C}$ parameters of the Dill equations.[1] More recently, the inclusion of refractive index changes [2] has led to an even more rigorous description. $[3,4]$ The second part of the image formation, the development step in which the latent image is transformed into the threedimensional relief structure, is much less understood from a theoretical point of view. The dissolution rate is generally expressed as a function of the sensitizer concentration $\mathrm{m}$, which may be directly obtained as a function of depth into the resist by a solution of the Dill equations. The procedures for the extraction of such data sets have recently been reviewed.[5] It is possible, and has indeed been done on occasion, to generate a lookup table for the dissolution rate $\mathbf{R}$ as a function of $\mathrm{m}$. However, due to the often considerable scatter involved in the dissolution rate data, such an approach requires piecewise smoothing of the $R$ data as a function of $m$ and may be quite laborious. It is therefore generally preferred to fit the data set to a functional dependence of $R$ on $m$, the so-called $R(m)$ curve.

The earliest models proposed for the $R(m)$ curve were designed to give a representation of the data without attaching an obvious physical significance to the fit parameters. An example of such an R(m) function is the Dill dissolution rate model:

$$
R(m)=\exp \left(E_{1}+E_{2} m+E_{3} m^{2}\right)
$$

The "Original Mack model"[6] can be derived from a consideration of the photolysis kinetics of multifunctional diazonaphthoquinone PACs [7]; provided that only the fully photolyzed PAC molecules lead to a dissolution promotion:

$$
\begin{gathered}
R(m)=R_{\max } \frac{(a+1)(1-m)^{n}}{a+(1-m)^{n}}+R_{\min } ; \\
a=\frac{n+1}{n-1}\left(1-m_{t h}\right)^{n}
\end{gathered}
$$

where $m_{t h}$ is the value of $m$ at the inflection point of the curve, and $\tilde{\mathbf{n}}$ is the developer selectivity. From the derivation, $\tilde{\mathbf{n}}$ is expected to be equal to the number of DNQ moieties in the PAC; in practice, it is often much high

The "Enhanced Mack model"[8] yields a superset of the Original Mack model curve family:

$$
\begin{gathered}
R(m)=R_{\text {resin }} \frac{1+k_{e n h}(1-m)^{n}}{1-K_{m h}(m)^{l}} ; \\
R_{m i n}=\frac{R_{r e s i n}}{1+k_{l n h}} ; R_{\max }=R_{r e s i n}\left(1+k_{e n h}\right)
\end{gathered}
$$

where $k_{\text {enh }}$ is the rate constant for the enhancement mechanism, $k_{\text {inh }}$ is the rate constant for the inhibition mechanism, $\tilde{n}$ is the enhancement reaction order, 1 is 
the inhibition reaction order, and $R$ resin is the dissolution rate of the resin. Its derivation is based on similar kinetic arguments but takes into account that dissolution promotion and inhibition branches may have different dissolution kinetics. The larger number of parameters leads to additional flexibility in the curve fitting.

For many conventional photoresists, all of the above models provide an adequate description of the $R(\mathrm{~m})$ curve, with the Enhanced Mack model being perhaps a first among peers. Many of the modern highperformance i-line resists, however, show a "notch" in the development rate curve at high sensitizer concentrations ( $m$ values) which is not adequately described by the currently employed development rate equations. This high-m notch is highly relevant to the imaging behavior of the resists because the region of low solubility is what determines the endpoint of the development process. A recent publication[8] has shown that the "notch" has a strong influence on the quality with which experimental results are reproduced by the simulation. It was shown that the reproduction of depth of focus, sidewall angles, and the definition of the tops of resist features all were significantly improved in the simulations if a manually edited parameter file with the "notch" was used instead of a standard dissolution rate equation. [9]

It is therefore desirable to have a development rate equation that can accurately describe also the high-m region. As will be shown below, such an equation can be derived from the equations governing the diffusion characteristics of the developer cations in the thin gel layer that forms at the resist/developer interface, the so-called penetration zone.

\section{The New Development Rate Model}

2.1. The Origin Of The "High-m Notch" In The Critical Concentration Phenomenon

Huang, Reiser and Kwei [10] have studied the diffusion characteristics of the developer in the penetration zone. It was found that the diffusion of the counter cations is the rate limiting factor, apparently since the cations must completely shed their hydration sphere before they can enter into the resin. This leads to a dramatic decrease of the diffusion coefficient $\mathrm{D}$ close to the penetration zone/resin interface; using the depth $\mathrm{x}$ into the penetration zone, this can be represented by $D=D_{0}(1-x)^{n /(n+1)}$. From this functional form, it is possible to derive an equation for the dependence of the dissolution rate on the developer concentration (Huang-Reiser-Kwei (HRK) equation) [10]:

$$
R=\frac{D}{\delta}=\frac{\alpha a^{n}}{\delta} \frac{n+2}{(n+1)^{2}} \frac{\left(c-c^{*}\right)^{n+1}}{\left(c+c^{*}\right)}
$$

where $\delta$ is the thickness of the penetration zone, and $\mathrm{c}^{*}$ is the critical concentration below which no development takes place. The value of $\mathrm{c}^{*} \mathrm{can}$ be determined by measuring dissolution rates in developers of varying strength and finding the concentration at which development ceases. The physical meaning of $n$ is given by $n /(n+1)$ being the exponent of the position dependence of the diffusion coefficient in the penetration zone.

The HRK equation or variations thereof [11] can be shown to very accurately describe the dependence of the dissolution rate of phenolic resins on the developer normality, whether they are novolak [11], polyhydroxystryene [12], or unexposed and exposed photoresist [13], with the latter two cases being the ones of interest here. In particular, the unexposed $(m=1)$ dissolution rate of many modem resists in standard $2.38 \%$ TMAH developer is zero, indicating that for these materials, $\mathrm{c}^{*}$ is higher than the developer concentration $(c=0.265 \mathrm{~N})$. If the resist is irradiated with increased doses, dissolution will occur at some point, i.e., $\mathrm{c}^{*}$ must be a function of the exposure state, or $c^{*}=c^{*}(m)$. The high-m end of the "notch" occurs at a sensitizer concentration $\mathrm{m}^{*}$, corresponding to the point between $0<\mathrm{m}<1$ where $\mathrm{c}^{*}$ first drops below the developer concentration, leading to a non-zero dissolution rate. For strongly exposed photoresist $\left(\mathrm{m}<<\mathrm{m}^{*}\right)$, very fast dissolution occurs, and $\mathrm{c}^{*}$ is certainly smaller than $0.265 \mathrm{~N}$.

From the above, it seems intuitively clear that the origin of the high-m behavior must lie in the phenomenon of cessation of development at the critical concentration, and that this can be adequately described by the HRK model. 


\subsection{Effects Of Exposure On c*}

To obtain a functional dependence of $\mathrm{c}^{*}$ on $\mathrm{m}$, rate measurements need to be done in more and more dilute developers for exposed and post exposure baked resists at various exposure doses. The PEB is necessary to remove or at least strongly attenuate the standing wave so that only slowly varying $m(z)$ values can be extracted from a simulation package such as PROLITH/2. Alternatively, the work can be carried out on a bottom antireflective coating to avoid standing waves altogether. From initial work, it was concluded that $\mathrm{c}^{*}(\mathrm{~m})$ can be described by a general S-shaped curve, the curvature of which determines the degree of non-linear behavior for high $\mathrm{m}$ values. As soon as $\mathrm{c}^{*}$ exceeds the developer concentration $\mathrm{c}$, the dissolution rate will in general become imaginary, and we will set $R(m)=0$ for $c^{*} \geq c$. The existence of a $c^{*}$ that is smaller than the developer concentration together with the nonlinearity of $c^{*}(m)$ causes the "notch" or "dropdown" at high $m$ values.

In a fit of an actual $R(m)$ curve, the following generalized S-shape functional relationship may be used for $c^{*}(m)$ :

$$
f(m)=D_{1}\left(1-\frac{1}{1+e^{D_{2}\left(m-D_{3}\right)}}\right)+D_{4}
$$

where the $D_{l}(\mathrm{i}=1$ to 4$)$ are adjustable parameters. An additional adjustable parameter used in the fit of the development rate is $D_{5}=\alpha \cdot a^{n} / \delta$, which determines the absolute height of the dissolution rate curve.

The value of $\mathbf{n}$ can be directly determined from the Huang-Reiser-Kwei plot according to

$$
\begin{aligned}
\frac{\partial \log \left(R\left(c+c^{*}\right)\right.}{\partial \log \left(c-c^{*}\right)} & =\left(c-c^{*}\right) \frac{\partial \log \left(R\left(c+c^{*}\right)\right)}{\partial c} \\
& =(n+1)\left(c-c^{*}\right) \frac{\partial}{\partial c} \log \left(c-c^{*}\right) \\
& =n+1
\end{aligned}
$$

It has been observed that $\mathbf{n}$ is a function of the copolymer composition in hydroxystyrene copolymers [12]. Initial experiments also showed $\mathbf{n}$ to be a function of $\mathrm{m}$, and the same general $\mathrm{S}$-shaped function as for $c^{*}(\mathrm{~m})$ above was used in the simulations.

\subsection{Tests Of The New Development Rate Equation}

The development rate equation was initially tested for the $A Z^{\mathbb{\Phi}} 7900$ high resolution i-line resist. Fig. 1 shows a graphical representation of the $R(\mathrm{~m})$ curve as fitted to the experimental data. The corresponding $c^{*}(\mathrm{~m})$ and $\mathrm{n}(\mathrm{m})$ curves are shown in Figs. 2 and 3.

Inserting the $c^{*}(m)$ curve into eq. (1) and optimizing the parameters by means of the Solver function in an Microsoft Excel ${ }^{\mathrm{TM}}$ spreadsheet gave an accurate fit of the experimental $\mathbf{A Z} \mathbf{Z}^{\oplus} 7900$ development rate curve for all $\mathrm{m}$ values, including the "notch" region just before and the region above $\mathrm{m}^{*}$. The $\mathrm{c}^{*}(\mathrm{~m})$ values may actually reach unreasonably high values for very high $\mathrm{m}$, but this has no impact on the fit since $R(m)=0$ for $c<c^{*}$. The optimization further shows that $n(m)$ is located on only the upper half of the S-shaped curve (Fig. 3), i.e., it is a constant for high $\mathrm{m}$ and shows a decrease only in the low $\mathrm{m}$ region. The reason for this behavior will be discussed in Section 2.4 .

Fig. 4 shows a comparison of the Dill, Original and Enhanced Mack to the new development rate model. It can be seen that the Original Mack and Dill models have a similar shape, and that the Enhanced

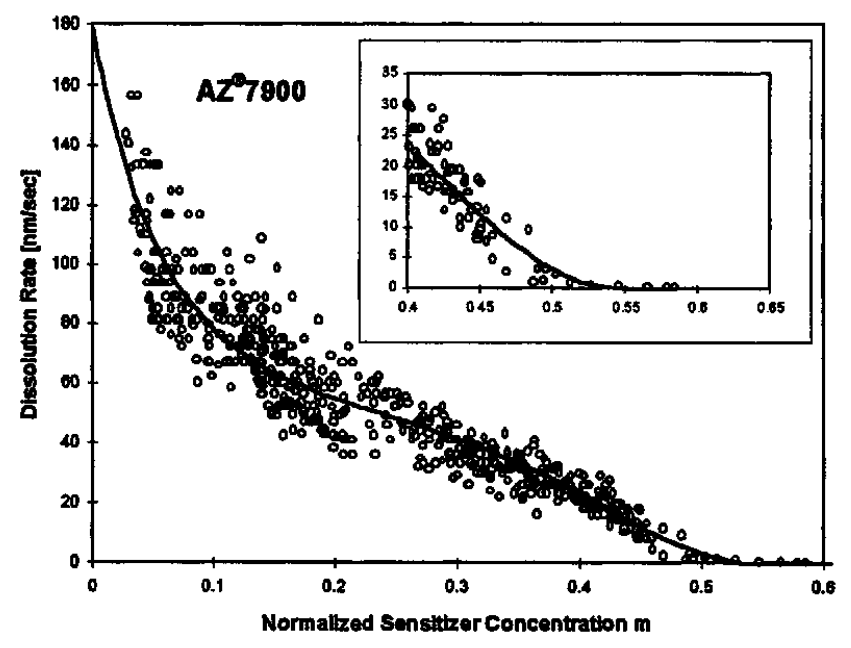

Fig. 1: Fit of the new development rate model to experimental data for the $A Z^{\infty} 7900$ high-resolution resist. 
Mack and the new model show comparable fit quality over large parts of the curve. Differences between the latter two are observed in the region of the "notch" and in the low-m region below $m=0.15$, where the new formalism allows higher flexibility of the curve. However, such low $\mathrm{m}$ is typically never observed in imagewise exposures.

In cases where $c$ is higher than $c^{*}$ in the range over which $R(m)$ curves are measured, eq. (4) predicts no "notch". Actually, a near-perfect fit of data calculated from a Dill or Original Mack development rate curve (both of which do not include the "notch") can be obtained by having a linear or constant $c^{*}(\mathrm{~m})$ curve.

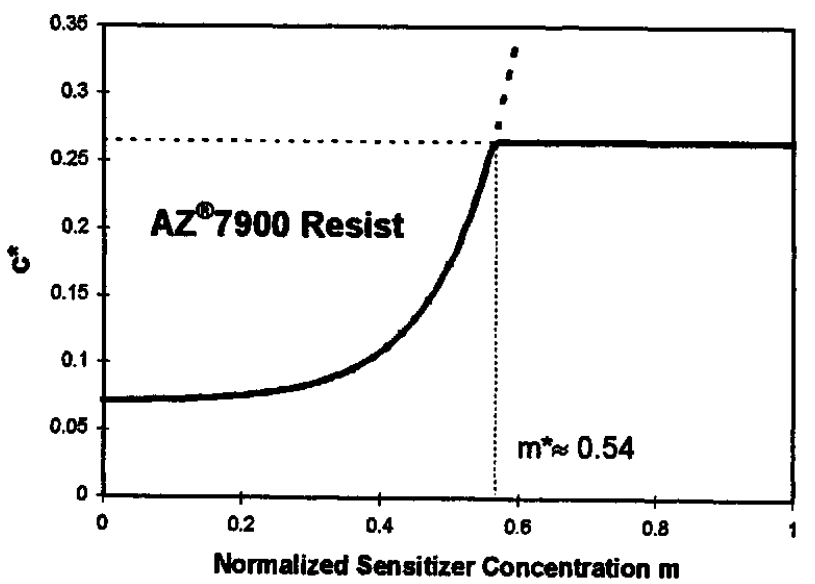

Fig. 2: Fitted $c^{*}(m)$ curve for $A Z^{\oplus} 7900$. Cessation of development is observed for an $\mathrm{m}^{*}$ value of 0.54 .

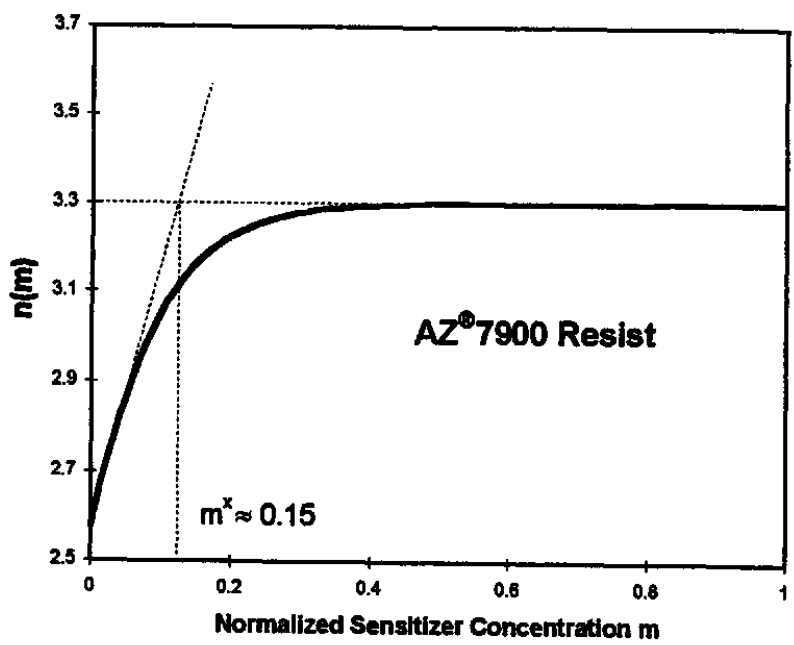

Fig. 3: Fitted $n(m)$ curve for $A Z^{\$} 7900$. The glass transition temperature of the penetration zone reaches the developer temperature for $23^{\circ} \mathrm{C}$ for $\mathrm{m}^{\mathrm{x}}=0.15$.
The $\mathrm{AZ}^{\circledast} 7200 \mathrm{i}$-line photoresist is a fast system optimized for high throughput applications. For this resist, the "notch" phenomenon is not observed, and the Original or Enhanced Mack models provide a satisfactory description of the dissolution rate. As can be seen from Fig. 8, there is no appreciable difference between the fits obtained for the four different models. The new development model predicts cessation of development for a value of $\mathrm{m}^{*}=0.93$; however, this is outside of the range of the present experimental data.
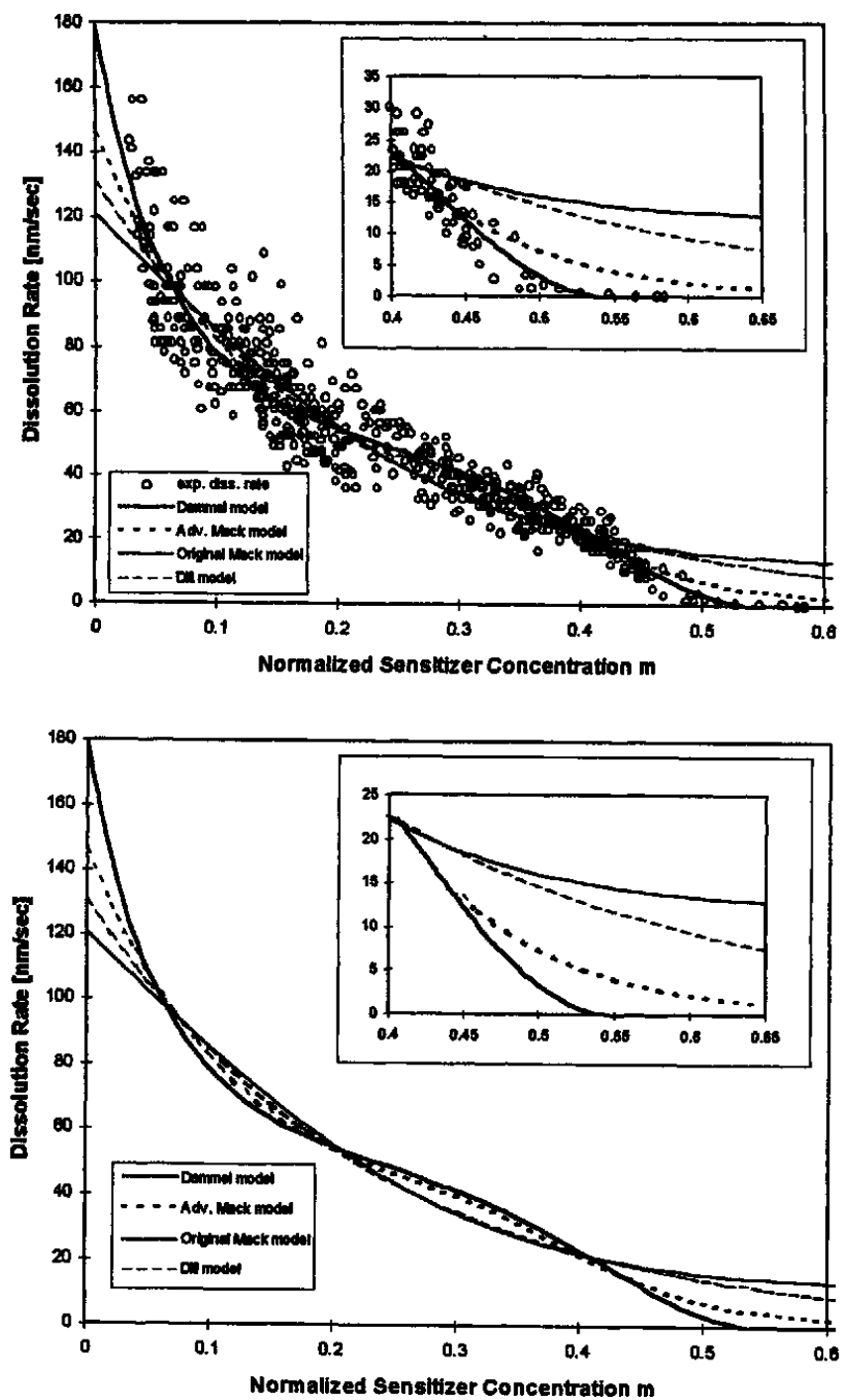

Figure 4: Comparison of existing $R(m)$ curve models and experimental data for $A Z^{8} 7900$ resist. The curves are shown again without exp. data points for clarity. 


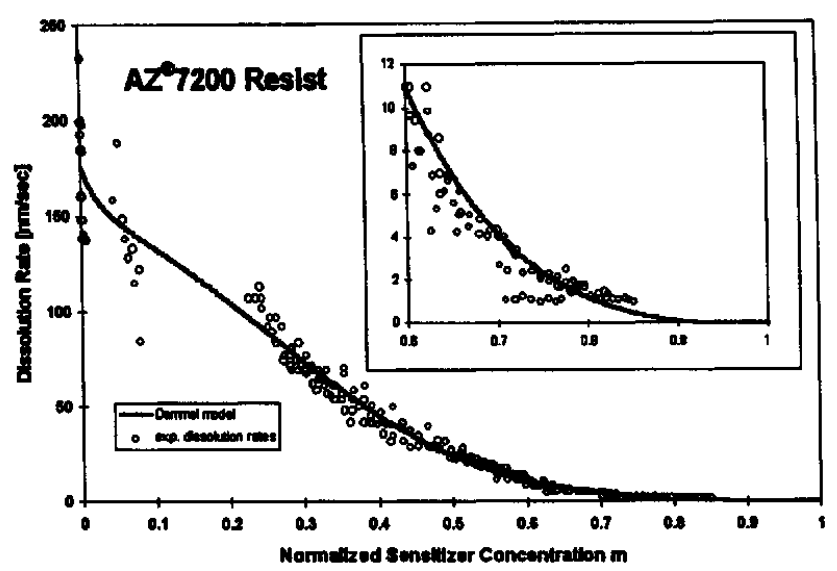

Fig. 5: Fit of the new development rate model to experimental data for the $\mathrm{AZ} Z^{\$} 7200$ high-speed resist.

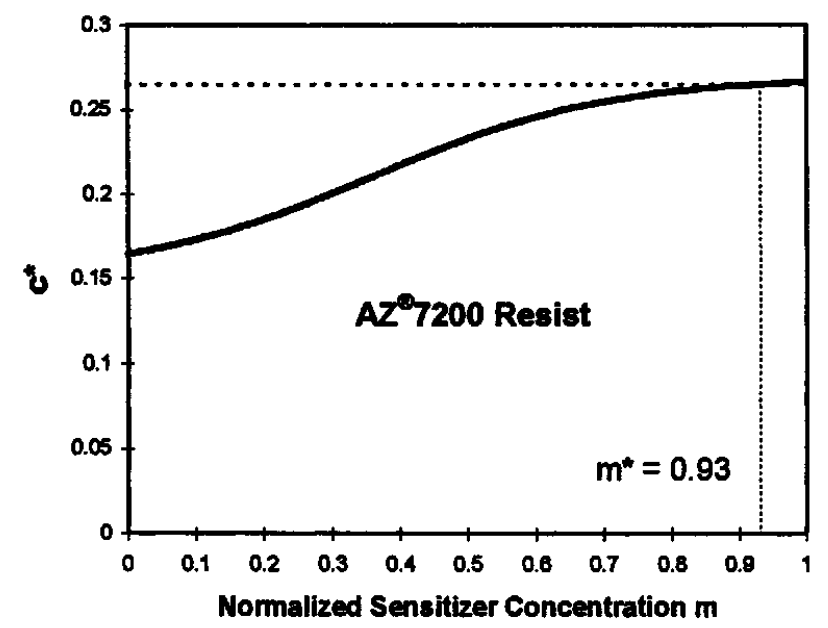

Fig. 6: Fitted $c^{*}(\mathrm{~m})$ curve for $A Z^{*} 7200$. Cessation of development is observed for an $\mathrm{m}^{*}$ value of 0.54 .

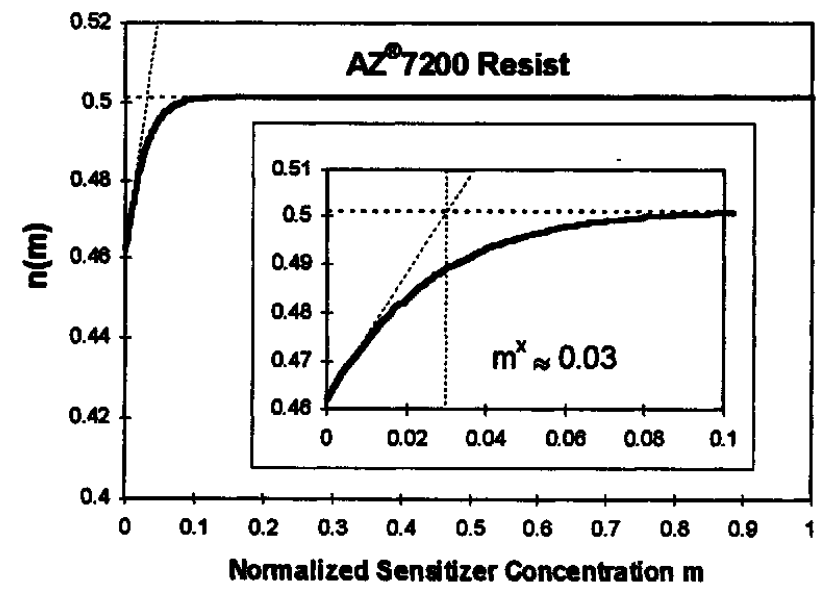

Fig. 7: Fitted $n(m)$ curve for $A Z^{\top} 7200$. The glass transition temperature of the penetration zone reaches the developer temperature for $23^{\circ} \mathrm{C}$ for $\mathrm{m}^{\mathrm{x}}=0.15$.
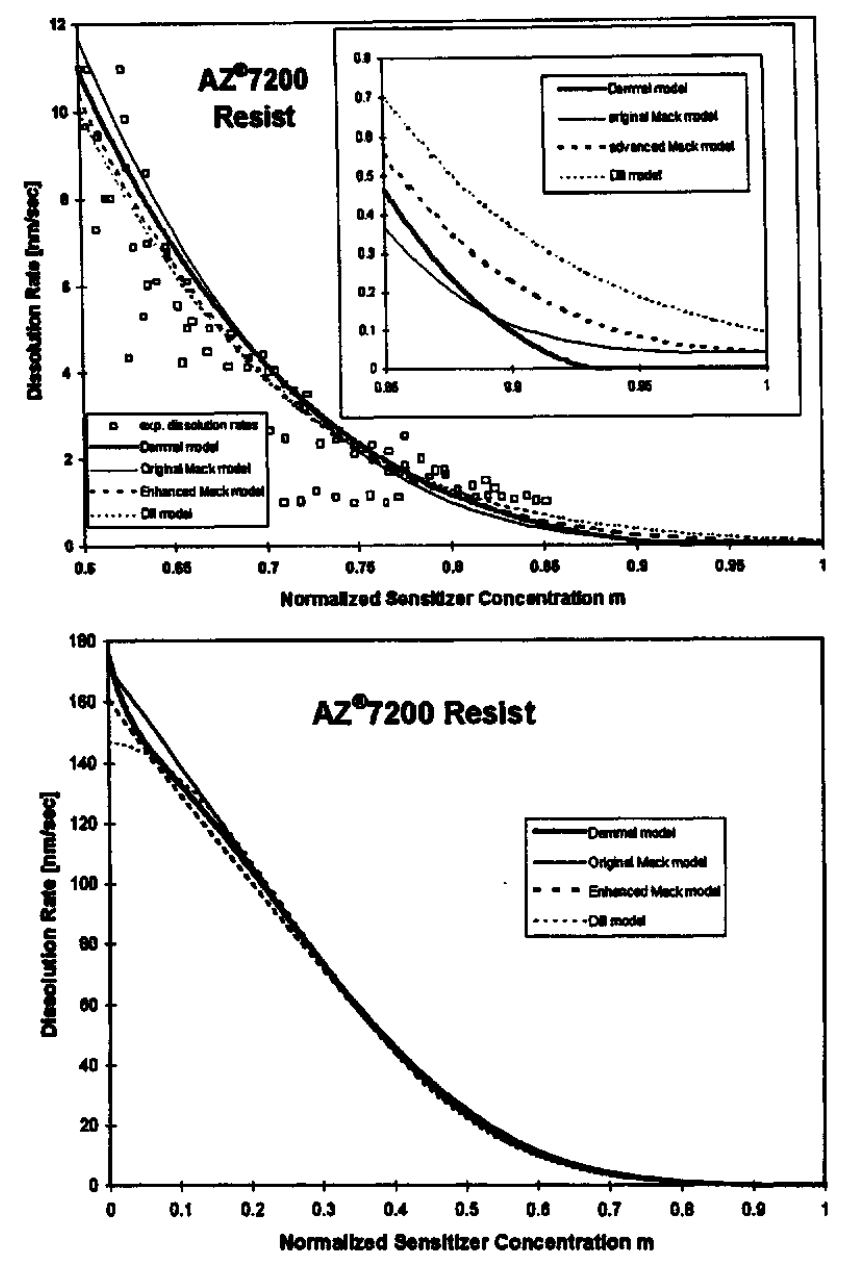

Figure 8: Comparison of existing $R(m)$ curve models and experimental data for $\mathrm{AZ}^{\$} 7200$ resist. The curves are shown again without exp. data points for clarity.

Table 1: Development Parameters for $\mathrm{AZ}^{\$} 7200$ and $A Z^{\circ} 7900$ resist.

\begin{tabular}{|l|c|c|c|c|}
\hline$A Z^{*} 7200$ & $D_{1}$ & $D_{2}$ & $D_{3}$ & $D_{4}$ \\
\hline$c^{*}(m)$ & 0.117861 & 5.742645 & 0.357259 & 0.151296 \\
\hline$n(m)$ & 0.285092 & 42.32072 & -0.04329 & 0.216368 \\
\hline$D_{5}$ & 1888.323 \\
\hline
\end{tabular}

\begin{tabular}{|c|c|c|c|c|}
\hline$A Z^{*} 7900$ & $D_{1}$ & $D_{2}$ & $D_{3}$ & $D_{4}$ \\
\hline$C^{*}(m)$ & 4.181069 & 10.13634 & 0.86201 & 0.070801 \\
\hline$n(m)$ & 3.298778 & 12.35013 & -0.10274 & 0.004005 \\
\hline$D_{5}$ & 3248.916 \\
\hline
\end{tabular}




\subsection{Physical Basis For The Change In n As A Function 0 f $m$}

Allowing $n$ to change according to Figs. 3 or 7 causes additional flexibility in the low-m, high- $R$ region of the curves. The decrease in $n$ leads to a rise in the dissolution rate corresponding well to the observed curves. A theoretical case for a drop in $\mathbf{n}$ consistent with the observed shape of the $n(m)$ curve can be made by inspection of the temperature and exposure dependence of the glass transition temperature of the penetration zone (Fig. 9). In the absence of neutral salts, cation transport in the penetration zone is the limiting factor for the development rate. When the glass transition temperature of the penetration zone is reached, the development mechanism changes from one in which cation transport occurs by percolation to one in which it occurs by polymer movement. DNQ sensitizers are inhibitors of percolation but not of polymer movement.[14] As the development mechanism gradually changes over to polymer movement above the $T_{B}, D N Q s$ will therefore cease to be dissolution inhibitors (this is why it is possible to have about $30-40 \%$ unexposed DNQ in parts of the film at the imaging dose).

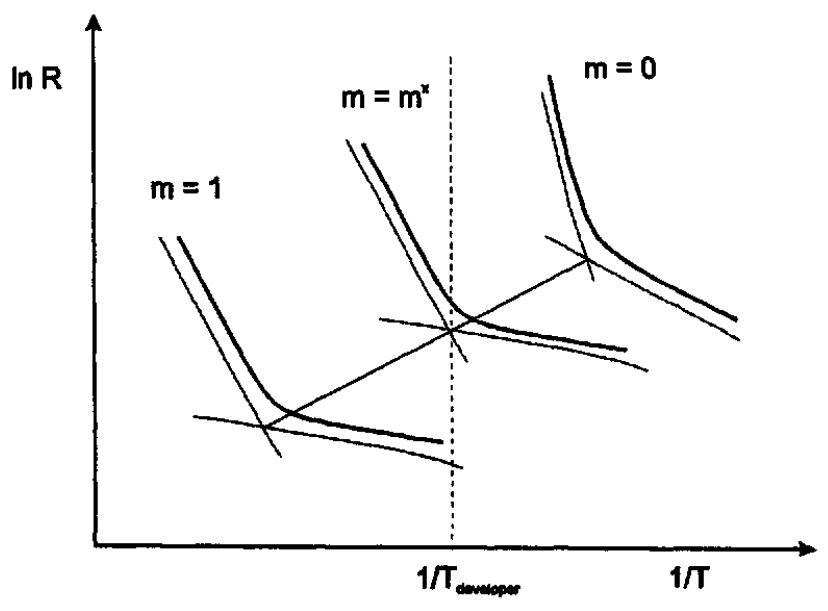

Fig. 9: Effects of the glass transition temperature of the penetration zone on the dissolution rate.

For unexposed resist, the glass transition temperature of the penetration zone is of the order of $43^{\circ} \mathrm{C}$, well above commonly used developer temperatures. However, for fully exposed resist, the $T_{g}$ can drop down to about $16^{\circ} \mathrm{C}$, well below commonly used developer temperatures. [14]

As the exposure progresses and $m$ drops, the crossover point will move along some trajectory from A to B. The exact shape of that trajectory is not known but there are reasons to believe that it will be linear. ${ }^{14}$ For some exposure, we will leave the lower branch and move over to the upper branch (point $\mathrm{C}$ at PAC concentration $\mathbf{m}^{\mathrm{x}}$ ). This point should coincide with the observed slope change in the $n(m)$ curve (Figs. 3 and 7). For $m>m^{x}, n$ will be approx. constant; and it will change to a new value of $n$ for $\mathbf{m} \ll \mathbf{m}^{\mathbf{x}}$. For the examples studied here we never reach the point at which $n$ attains the new value but remain in the transition zone even for $\mathbf{m}=0$.

\section{Conclusions}

It has been shown that a new development rate equation based on the Huang-Reiser-Kwei treatment of diffusion in the penetration zone accurately describes experimental $R(m)$ curves if the parameters $\mathrm{c}^{*}$ and $\mathrm{n}$ are taken to be functions of the sensitizer concentration $\mathrm{m}$. For high resolution resists (exemplified here by $A Z^{\circledR} 7900$ ), both the "notch" phenomenon at high $\mathrm{m}$ as well as the more than linear dissolution rate increases for low $m$ are reproduced better than with the standard dissolution rate models used today. For conventional resists (e.g., $A Z^{\circledR} 7200$ ), the fit of the new model to the curve is essentially indistinguishable from that obtained with the Enhanced Mack model.

The fit functions used for $c^{*}(m)$ and $n(m)$ were generalized $S$ curves, requiring 4 parameters each. Another parameter is required to determine the absolute value of the dissolution rate, bringing the total number to nine parameters per photoresist. It appears possible that this rather large number of parameters may be reduced if the underlying phenomena for the $c^{*}(m)$ and $n(m)$ behavior can be stated in a mathematical form.

All parameters in the new development rate equation have physical meaning. It should in principle be possible to determine $c^{*}(\mathrm{~m})$ and $\mathrm{n}(\mathrm{m})$ by dissolution rate measurements at different developer strengths, 
and then to predict the $R(m)$ curve values except for a scaling factor given by the parameter $D_{5}$. Such a prediction of the $R(m)$ curve would be a strong argument for the physical validity of the new model. From a more practical viewpoint, the new equation should be helpful in providing a phenomenologically correct description of the high-m behavior of photoresists.

\section{References}

1) F.H. Dill, W.P. Homberger, P.S. Hauge, and J.M. Shaw, IEEE Trans. Electron Devices 22(4), 445-452 (1975).

2) C.L. Henderson, C.G. Willson, R.R. Dammel, and R.A. Synowicki, Proc. SPIE 3059 (1997), in print.

3) A. Erdmann, C.L. Henderson, C.G. Willson, and W. Henke, Proc. SPIE 3059, (1997), in print.

4) A. Erdmann, C.L. Henderson, C.G. Willson, and W. Henke, Proc. SPIE 3059, (1997), in print.

5) C.L. Henderson, S.N. Pancholi, S.A. Chowhury, C. G. Willson, R.R. Dammel, Proc. SPIE 3059, (1997), in print; C.L. Henderson, P.C Tsiartas, S.N. Pancholi, S.A. Chowhury, C. G. Willson, K.D Dombrowski, R.R. Dammel, Proc. SPIE 3059, (1997), in print.
6) C. Mack, J. Electrochem.Soc. 134(1), 148-152 (1987)

7) P. Trefonas and B.K. Daniels, Proc. SPIE $\underline{\mathbf{7 7 1}}$, 194-210 (1987); P. Trefonas and C. Mack, Proc. SPIE1466, 117-131 (1991)

8) C. Mack, J. Electrochem. Soc. 139(4), L35-L37 (1992); C.A. Mack, "Inside Prolith TM", Finle Technology, Austin, TX 1997, ISBN 09650922-0-8, p. 106ff.

9) G. Arthur, B. Martin, and C.A. Mack, Proc. SPIE 3059 (1997), in print.

10) J.-P. Huang, T.K. Kwei and A. Reiser, Proc. SPIE 1086, 74 (1989); Macromolecules 22. 4106 (1989).

11) T.F. Yeh, H.Y. Shi, A. Reiser, M.A. Toukhy, and B.T. Beauchemin, Jr., J. Vac. Sci. Technol. B 10, 715 (1992).

12) R. Dammel, M.D. Rahman, P.H. Lu, and V. Elango, Polym. Adv. Technol. $\underline{5}$ (1), 28-40 (1994); R.R. Dammel, M.D. Rahman, P.H. Lu, A. Canize, and V. Elango, Proc. SPIE 2195, 542-557 (1994).

13) R.R. Dammel, unpublished results.

14) A. Reiser, H.-Y. Shi, T.-F. Yeh, and J.-P. Huang, Angew. Chem. Intl. Ed. 35(21), 24132548 (1996). 